\title{
Mössbauer Studies of Fusion Crust of the Soltmany Meteorite
}

\author{
Ł. Karwowski ${ }^{a}$, K. Brzózka ${ }^{b, *}$, T.A. Przylibski ${ }^{c}$, P. Duda ${ }^{d}$, B. Górka $^{b}$, \\ M. GAWroński ${ }^{b}$, M. JAKUBOWsKa ${ }^{d}$ AND K. LUSZCZEK $^{c}$ \\ ${ }^{a}$ University of Silesia in Katowice, Faculty of Earth Sciences, Department of Geochemistry, \\ Mineralogy and Petrology, Będzińska 60, 41-200 Sosnowiec, Poland \\ ${ }^{b}$ University of Technology and Humanities, Faculty of Mechanical Engineering, Department of Physics, \\ E. Stasieckiego 54, 26-600 Radom, Poland \\ ${ }^{c}$ Wrocław University of Science and Technology, Faculty of Geoengineering, Mining and Geology, \\ Division of Geology and Mineral Waters, Wybrzeże S. Wyspiańskiego 27, 50-370 Wrocław, Poland \\ ${ }^{d}$ Warsaw University of Technology, Faculty of Physics, Koszykowa 75,00-662 Warsaw, Poland \\ Studies of the fusion crust of a meteorite allow to understand the processes occurring during its flight through \\ the Earth atmosphere. Transmission Mössbauer spectroscopy has been used to determine iron-bearing phases \\ present in the fusion crust of meteorite Sołtmany. The test material was obtained by mechanical detachment \\ followed by grinding. The results reveal that the mineral composition of the fusion crust is close to the mineral \\ composition of the meteorite interior, i.e. it comprises olivine, pyroxene, kamacite, and troilite. However, antitaen- \\ ite and taenite with a partially disordered structure were also found in the fusion crust.
}

DOI: 10.12693/APhysPolA.134.1076

PACS/topics: 96.30.Za, 91.65.Sn, 75.50.Bb, 76.80.+y, 33.45.+x

\section{Introduction}

The Sołtmany meteorite fell on April 30th, 2011 at 6:03 am and was found a few minutes thereafter. This chondrite is the 9th witnessed fall of a meteorite in Poland and represents the petrographic type L6. The shock stage is defined as S2 and the weathering grade is W0 [1]. As a fresh fall Soltmany is covered with a black, darkbrownish, and somewhat shiny fusion crust. The surface texture of the crust is slightly rough, and it exhibits small vesicles. Under a magnifying glass, it is shiny and glassy. The thickness of the fusion crust was measured on cut sections and it varies slightly from 0.5 to $0.7 \mathrm{~mm}[1,2]$. The Soltmany chondrite is the most widely studied Polish meteorite [1-11].

Phase composition of the fusion crust, based on the macro- and microobservations showed that the most external part is opaque and semi-translucent. It is mainly composed by amorphous material, which contains closed pores. Their chemical composition is dominated by silicates containing Fe-rich feldspar. Directly under the external layer olivine relics and small pyroxene crystals are present. The semi-translucent, brown to yellow pyroxenes form radial aggregates containing $\mathrm{Fe}$, which is responsible for their color. The amorphous material fills interstitial space between the olivine and pyroxene crystals. In the so-called black veins zone, numerous veins of metallic phases (Ni-rich) occur. Occasionally, troilite veins are associated with the metallic phases. In the most external, amorphous zone of the fusion crust, a little crystallites of $\mathrm{Fe}-\mathrm{Ni}$ spinel were noted (trevorite) [1].

\footnotetext{
*corresponding author; e-mail: k.brzozka@uthrad.pl
}

The goal of presented studies is more precise determination of iron bearing phases of the fusion crust by means of the Mössbauer spectroscopy and the comparison of the mineral composition of the fusion crust with that of the meteorite interior. Moreover, studies of fusion crust allow to understand the processes occurring during the meteorite flight through the Earth atmosphere which is the last process forming the mineral composition of the meteorite in its surface part.

\section{Experimental details}

Fragments of the fusion crust for the Mössbauer studies were obtained by mechanical detachment using steel needles and blades. This work was done manually by observing a sample of chondrite using a stereoscopic optical microscope. Then the fragments of the fusion crust were ground using a ball mill. Aluminum oxide was used as an abrasive material. The last process of preparing the material for the Mössbauer studies was an attempt of magnetic separation of powdered fusion crust material in order to thorough investigation of magnetic phases, while in most ordinary chondrites non-magnetic phases are prevailing. This separation was partly successful, so only small amount of the material enriched with magnetic fraction was obtained. Due to the large number of very fine magnetic grains of the $\mathrm{Fe}-\mathrm{Ni}$ alloy, silicates grains (containing: olivine, pyroxene and plagioclase) with a small even $\mathrm{Fe}-\mathrm{Ni}$ grain content were also separated as magnetic fraction. As a result, two samples of the fusion crust were prepared. One, very small, with a significant content of the magnetic fraction (sample A) and the other, in which there were mainly non-magnetic grains (sample B). 
In order to identify iron bearing phases present in the fusion crust and determine their relative contribution, ${ }^{57} \mathrm{Fe}$ based Mössbauer spectroscopy was used. Transmission measurements were performed by a spatial arrangement with vertical direction of gamma beam. In order to modify the radiation energy, the Doppler effect was employed; a source of radiation was placed on a shaft of vibrator moving longitudinally in a constant acceleration mode.

In the case of the sample A (magnetically enriched fraction), due to very small amount of the investigated material, the Mössbauer measurement lasted several weeks. In such a situation effect of iron contamination present in a beryllium window of the gas proportional counter used as a detector can reveal itself. As it turned out, in our measurement this effect also took place. In order to test the window purity, a comparative experiment was performed without any absorber, in which the Mössbauer spectrum originated only from vestigial amount of iron present in the counter window.

The Mössbauer spectra were analyzed by means of the Normos program using least-square procedure for fitting lines or their groups (i.e. doublets or sextets) with the Lorentzian profile. The constant ratio of the spectral areas of individual lines belonging to the Zeeman sextets $A_{1,6}: A_{2,5}: A_{3,4}=3: 2: 1$ (where $A_{1,6}$ is related to line 1 or 6 , etc.) was assumed.

\section{Results and discussion}

The Mössbauer spectrum of sample A, presented in Fig. 1, takes a complex form. Hyperfine interactions parameters of components: isomer shift $\delta$ (in relation to pure iron), quadrupole interaction parameter (quadrupole shift or quadrupole splitting) $\Delta$ and hyperfine magnetic field $B$ and also spectral area $A$ were derived in the course of the fitting procedure. A sextet of hyperfine magnetic field $33.5 \mathrm{~T}$ and other hyperfine interactions parameters close to zero dominates in the spectrum. More accurate analysis showed that the sextet is accompanied by a second one, with slightly smaller hyperfine magnetic field $29.2 \mathrm{~T}$ and quadrupole shift $0.23 \mathrm{~mm} / \mathrm{s}$. These two components are attributed to metallic phases in a form of $\mathrm{Fe}-\mathrm{Ni}$ alloy with different concentration of $\mathrm{Ni}$ and possible admixture of $\mathrm{Co}$ atoms. The former sextet is related to bcc $\mathrm{Fe}-\mathrm{Ni}$ with about $10 \%$ of $\mathrm{Ni}$, i.e. kamacite, while the latter one is ascribed to partially disordered taenite, i.e. fcc $\mathrm{Fe}-\mathrm{Ni}$ alloy with about $50 \%$ of $\mathrm{Ni}$ [12]. The third sextet is characterized by similar value of hyperfine magnetic field $(B=30.7 \mathrm{~T})$ but clearly larger isomer shift $=0.80 \mathrm{~mm} / \mathrm{s}$ and quadrupole shift $\Delta=1.02 \mathrm{~mm} / \mathrm{s}$, the values typical of non-magnetic iron sulfide-troilite (FeS). Moreover, the spectrum comprises, in a small amount, a doublet characteristic of olivine. Owing to the fact that the spectrum is rather smeared, these two components were extracted in such a way that their hyperfine interactions parameters $\delta, \Delta, B$ were fixed and afterwards the values of spectral

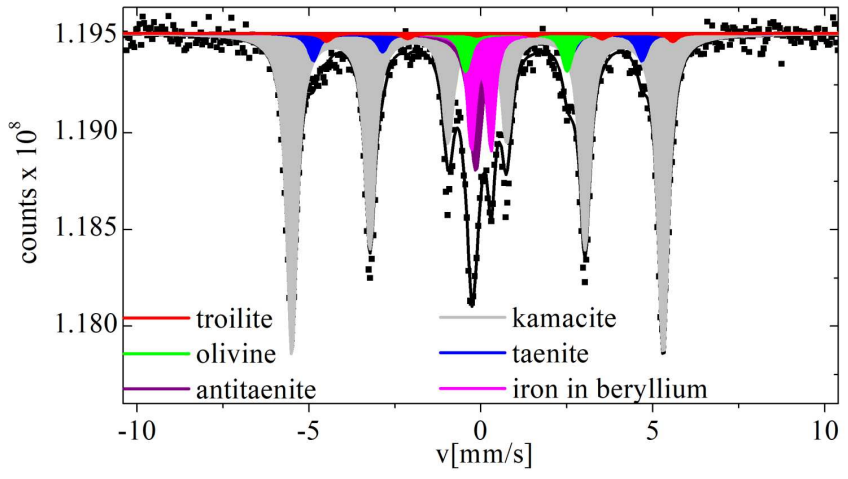

Fig. 1. Mössbauer spectrum of sample A (enriched in magnetic phases) of the Soltmany chondrite fusion crust.

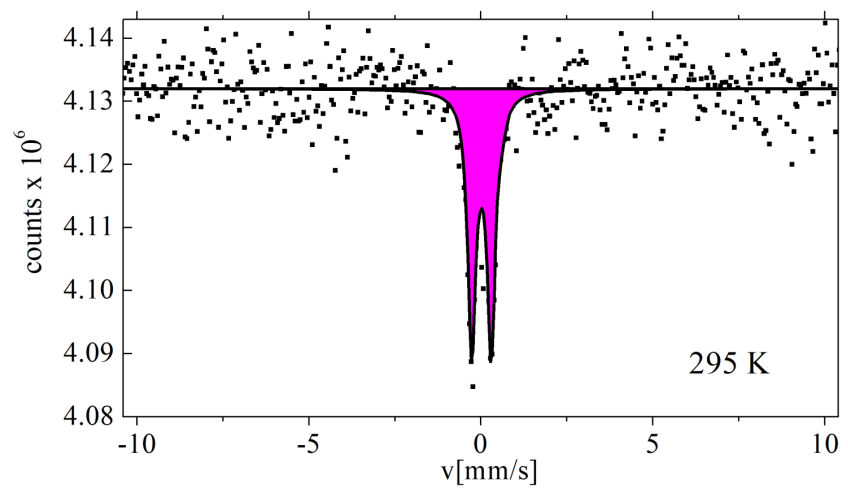

Fig. 2. Mössbauer spectrum collected during the test of purity of the proportional counter window which evidenced a doublet related to iron in beryllium.

area were fitted. In the central part of the spectrum an extra doublet is visible, coming from iron traces present in the counter window made of beryllium. The values of its isomer shift $\delta=0.12 \mathrm{~mm} / \mathrm{s}$ and quadrupole splitting $\Delta=0.58 \mathrm{~mm} / \mathrm{s}$ are in good agreement with those derived from the spectrum collected during the test of purity of the window, which is presented in Fig. $2(\delta=0.12 \mathrm{~mm} / \mathrm{s}$, $\Delta=0.59 \mathrm{~mm} / \mathrm{s})$. This doublet is identified as belonging to iron-in-beryllium solid solution, therefore it is not related to any constituents of the investigated material. Hyperfine interactions parameters of the components, determined from the spectra, are collected in Table I. Relative spectral areas $p$ were calculated taking into consideration all the constituents. Later, the doublet attributed to iron in beryllium was ignored and the relative spectral areas $p^{*}$ were determined excluding this component.

About $10 \%$ of spectrum constitutes a broadened singlet with isomer shift $-0.05 \mathrm{~mm} / \mathrm{s}$. It is attributed to antitaenite, i.e. low-Ni paramagnetic taenite (with approximately $25 \%$ of $\mathrm{Ni}$ ). This phase was found in some ordinary chondrites (for instance see $[12,13]$ ) and, like other phases of taenite, was formed during very slow and long cooling of the meteorites. 
Hyperfine interactions parameters of the components of the Mössbauer spectrum collected for the sample A (enriched in magnetic phases) of the Soltmany chondrite fusion crust: $\delta$ - isomer shift in relation to bcc Fe, $\Delta-$ quadrupole interaction parameter, $B$ - hyperfine magnetic field, $G$ - full width at half maximum, $p$ - relative spectral area, $p^{*}$ - relative spectral area excluding the doublet attributed to iron in beryllium.

\begin{tabular}{l|c|c|c|c|c|c|c}
\hline \hline & $\delta[\mathrm{mm} / \mathrm{s}]$ & $\Delta[\mathrm{mm} / \mathrm{s}]$ & $B[\mathrm{~T}]$ & $G[\mathrm{~mm} / \mathrm{s}]$ & $\Theta\left[{ }^{\circ}\right]$ & $p[\%]$ & $p^{*}[\%]$ \\
\hline kamacite & 0.01 & 0.01 & 33.5 & 0.37 & - & 69.6 & 76.7 \\
taenite (partially disordered) & -0.02 & 0.23 & 29.2 & 0.38 & - & 6.6 & 7.3 \\
troilite & 0.80 & 1.02 & 30.2 & 0.37 & 61.5 & 1.0 & 1.1 \\
olivine & 1.14 & 2.95 & - & 0.36 & - & 3.2 & 3.6 \\
antitaenite & -0.05 & 0 & - & 0.46 & - & 10.2 & 11.3 \\
iron in beryllium & 0.12 & 0.58 & - & 0.27 & - & 9.4 & 0
\end{tabular}

The analysis of the Mössbauer spectra of the magnetically enriched fraction made possible to determine the presence of various minerals, both magnetic and nonmagnetic, in the fusion crust. This is due to the limited efficiency of magnetic separation of the powdered sample. It is connected with the presence of $\mathrm{Fe}-\mathrm{Ni}$ grains of very small size (about a few up to few-dozen of $\mu \mathrm{m}$ in diameter), which are inclusions in silicates and intergrowths with iron sulphide (FeS - troilite). Therefore, it was not possible to analyze the pure magnetic fraction. The obtained spectrum allowed to identify mineral phases whose presence in the fusion crust of the Soltmany chondrite was already known. These are: kamacite, taenite, troilite, and olivine. It is interesting to note the presence of antitaenite which, however, is not officially recognized as a mineral by the International Mineralogical Association. There were no new mineral phases identified, that may have formed during ablation melting and recrystallization of the fusion crust during the meteorite flight through the Earth atmosphere. Also vitreous phases, including plagioclase glass were invisible for the applied method. However, the presence of taenite with a partially disordered structure was stated, which may indicate that these grains experienced the first effects of temperature increase, which in the long term would lead to their complete melting.

Former Mössbauer investigations of the powdered sample coming out from meteorite Sołtmany [11] showed that doublets related to olivine (59.5\% of the spectral area) and pyroxene $(26.0 \%)$ provided the greatest contribution to the Mössbauer spectrum, while magnetic components - the Zeeman sextets attributed to kamacite (4.5\%) and troilite $(10.0 \%)$ - made the other part. The analysis of the Mössbauer spectrum of sample B (Fig. 3, Table II) exhibited that the spectral areas of its components were as follows: $63.7 \%$ of olivine, $19.5 \%$ of pyroxene, $0.6 \%$ of kamacite, and $15.2 \%$ of troilite. A comparison of the above results confirm a similar (but not identical) mineral composition and proportions of the mineral content of the low-magnetic fraction of the Sołtmany chondrite fusion crust to the mineral composition and proportion of mineral content of the Soltmany chondrite interior. Olivine was found in a very similar amount. However, the differences in the content of pyroxene (smaller), troilite (larger) and kamacite (smaller) in relation to the interior of the Sołtmany chondrite were observed. The two last discrepancies are most likely due to the depletion of sample B in the magnetic grains of kamacite and therefore relative enrichment in troilite (which is antiferromagnetic).

In the Mössbauer spectra of the low-magnetic fraction of the fusion crust also $\mathrm{Fe}^{3+}$ has been found, which is present in the composition of iron oxides or hydroxides created at the expense of $\mathrm{Fe}-\mathrm{Ni}$ grains in the chondrite weathering on Earth. It is natural that these processes first of all proceed at the surface of meteorite.

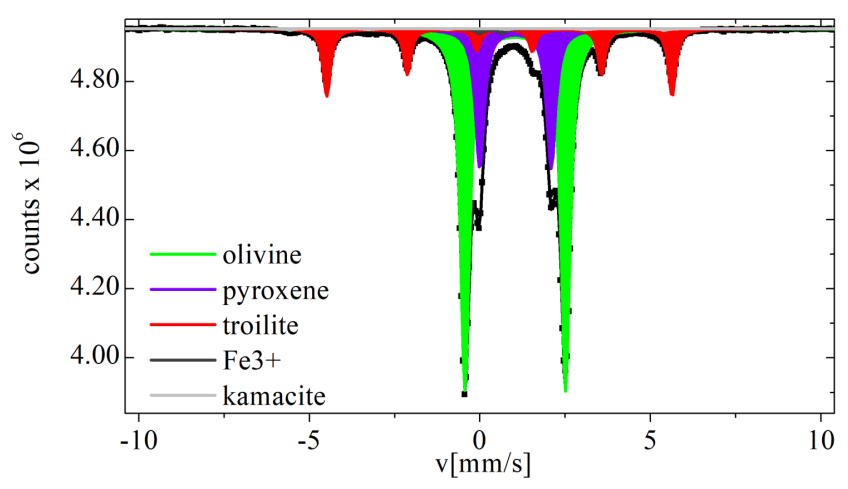

Fig. 3. Mössbauer spectrum of sample B (lowmagnetic fraction) of the Sołtmany chondrite fusion crust.

TABLE II

Hyperfine interactions parameters of the components of the Mössbauer spectrum collected for the sample B (low-magnetic fraction) of the Soltmany chondrite fusion crust: $\delta$ - isomer shift in relation to bcc Fe, $\Delta-$ quadrupole interaction parameter, $B$ - hyperfine magnetic field, $G$ - full width at half maximum, $p$ - relative spectral area, $p_{\text {int }}$ - relative spectral area obtained for interior of the Soltmany chondrite [11].

\begin{tabular}{l|c|c|c|c|c|c|c}
\hline \hline & $\begin{array}{c}\delta \\
{[\mathrm{mm} / \mathrm{s}]}\end{array}$ & $\begin{array}{c}\Delta \\
{[\mathrm{mm} / \mathrm{s}]}\end{array}$ & $\begin{array}{c}B \\
{[\mathrm{~T}]}\end{array}$ & $\begin{array}{c}G \\
{[\mathrm{~mm} / \mathrm{s}]}\end{array}$ & $\begin{array}{c}\Theta \\
{\left[{ }^{\circ}\right]}\end{array}$ & $\begin{array}{c}p \\
{[\%]}\end{array}$ & $\begin{array}{c}p_{\text {int }} \\
{[\%]}\end{array}$ \\
\hline kamacite & 0.02 & 0.01 & 33.9 & 0.27 & - & 0.6 & 4.5 \\
troilite & 0.76 & 1.00 & 30.9 & 0.27 & 61.7 & 15.2 & 10.0 \\
olivine & 1.14 & 2.95 & - & 0.30 & - & 63.7 & 59.5 \\
pyroxene & 1.15 & 2.09 & - & 0.30 & - & 19.5 & 26.0 \\
$\mathrm{Fe}^{3+}$ & 0.43 & 0.67 & - & 0.50 & - & 1.0 & -
\end{tabular}




\section{Conclusions}

Ablation melting occurs in the narrow outer zone of the meteorite. The thermal conductivity of chondrite rock is very low and in the short time of meteorite passage through the atmosphere causes that the main process is the melting of matter manifested by the formation of a porous glassy phase (glass with gas bubbles), leading then to complete evaporation. Even in the deeper layer of the fusion crust, no significant recrystallization processes occur, that could cause creation of new mineral phases. As previous research has shown [1] in this deeper zone of the fusion crust there are only phases with a disturbed, non-stoichiometric composition. However, these are chemically and probably also structurally modified minerals, which are present in the composition of the chondrite before entering the Earth atmosphere. This is confirmed by the presence of taenite grains with a partially disordered structure registered by the authors in the Mössbauer spectrum of the analyzed magnetic fraction of the fusion crust. The authors did not find any new mineral phases in the fusion crust of the Soltmany chondrite. The mineral composition of the fusion crust is practically the same as the mineral composition of the meteorite interior, i.e. it comprises olivine, pyroxene, kamacite, taenite, troilite, and antitaenite found for the first time in the Soltmany chondrite. The difference is marked only in the presence of glass and partially melted minerals with non-stoichiometric composition that is demonstrated by the presence of taenite grains with a partially disordered structure registered with the Mössbauer spectrum analysis. Small disparities in spectral areas of the components of the Mössbauer spectra can arise both from different ways of sample preparation and inhomogeneity of the content of the investigated object.

\section{References}

[1] Ł. Karwowski, Meteorites 2, 15 (2012).

[2] T.A. Przylibski, Acta Soc. Metheor. Pol. 7, 93 (2016).

[3] B. Woźniak, M. Woźniak, Meteorites 2, 9 (2012).

[4] T.A. Przylibski, K. Łuszczek, Meteorites 2, 31 (2012).

[5] K. Łuszczek, N. Dalcher, I. Leya, Meteorites 2, 39 (2012).

[6] M. Laubenstein, A. Giampaoli, P. Janowski, J.W. Mietelski, Meteorites 2, 45 (2012).

[7] M. Szurgot, R.A. Wach, T.A. Przylibski, Meteorites 2, 53 (2012).

[8] P. Rochette, J. Gattacceca, M. Lewandowski, Meteorites 2, 67 (2012).

[9] J. Gałąka-Friedman, K. Szlachta, Meteorites 2, 73 (2012).

[10] P. Schmitt-Kopplin, M. Harir, B. Kanawati, D. Tziozis, N. Hertkorn, Z. Gabelica, Meteorites 2, 79 (2012)

[11] J. Gałązka-Friedman, K. Szlachta, Ł. Karwowski, M. Woźniak, Hyperfine Interact. 226, 593 (2014).

[12] Y.A. Abdu, Y.A. Ericsson, T. Annersten, H. Dubrovinskaia, N.A. Dubrovinsky, L.S. Gismelseed, Hyperfine Interact. C 5, 375 (2002).

[13] A. Wojnarowska, T. Dziel, J. Gałązka-Friedman, Ł. Karwowski, Hyperfine Interact. 186, 167 (2008). 